\title{
Naturally Derived Photoinitiators for Dental and Biomaterials Applications
}

\author{
Mohamed Mahmoud Abdul-Monem \\ ${ }^{1}$ Dental Biomaterials Department, Faculty of Dentistry, Alexandria \\ University, Alexandria, Egypt
}

Eur Dent Res Biomater J 2020;1:72-78

\begin{abstract}
Address for correspondence Mohamed M. Abdul-Monem, BDS, MSc, PhD, Dental Biomaterials Department, Faculty of Dentistry, Alexandria University, Alexandria 21526, Egypt (e-mail: mohamed.mahmoud@dent.alex.edu.eg).
\end{abstract}

\begin{abstract}
Biocompatibility of materials used in dental and biomaterials applications is very important and depends on the components of these materials. Photopolymerized materials for dental and biomaterials applications have been progressively used since the 1970s. One of the crucial components in these materials is the photoinitiator ( $\mathrm{PI})$ that initiates the polymerization reaction. Synthetic Pls are the most commonly used types, but owing to their drawbacks such as cytotoxicity, insolubility in water, and high cost, research on naturally derived (bio-sourced) Pls is growing, to find an alternative to these synthetic types, especially in the growing field of three-dimensional (3D) printing and bioprinting of biomaterials for tissue engineering applications. Naturally derived Pls are biocompatible, highly water-soluble, and abundant. Naturally derived Pls have been used to prepare experimental dentine bonding agents, dentine primers, photo-crosslinked hydrogels for tissue engineering applications, antibacterial coatings, guided tissue regeneration membranes, and 3D printed biomaterials. An electronic search was done using MEDLINE/PubMed and Scopus databases using the keywords naturally derived, bio-sourced, photoinitiators dental, biomaterials, 3D printing, and 3D bioprinting, to review potential naturally derived Pls for dental and biomaterials

Keywords

- naturally derived

- bio-sourced

- photoinitiators

- dental

- biomaterials

- 3D printing

$-3 \mathrm{D}$ bioprinting applications. There are a variety of naturally derived PIs with various colors and absorption spectra to choose from, according to the intended application. Most of naturally derived PIs can be used with modern conventional dental light curing units, making them applicable for experimental studies for potential dental and biomaterials applications. Due to their biocompatibility and availability it is expected that in the upcoming years, research on naturally derived PIs and their dental and biomaterials applications will increase especially in the growing field of 3D bioprinting in which cell viability is essential; thus this review was done.
\end{abstract}

\section{Introduction}

Photopolymerization is a process in which a liquid monomer changes into a solid material by a chain reaction initiated by reactive species (radicals, cations, or anions), which are generated from photoinitiators (PIs), upon ultraviolet (UV) or visible light irradiation. ${ }^{1}$ Photopolymerization has been used in dentistry since the 1970s. It started with the use of a UV

DOI https://doi.org/ 10.1055/s-0040-1721313. light-cured pits and fissure sealant, ${ }^{2}$ then UV-cured dental resin-based composites (RBCs). However, due to the drawbacks of UV-light curing such as short depth of cure, long curing time per increment, heat generation, and potential hazards to the eyes, ${ }^{3}$ visible light-cured (RBCs) progressively replaced UV-cured (RBCs) after the introduction of camphorquinone PI in $1978 .{ }^{4}$

(c) 2020. European Dental Research and Biomaterials Journal.

This is an open access article published by Thieme under the terms of the Creative Commons Attribution-NonDerivative-NonCommercial-License, permitting copying and reproduction so long as the original work is given appropriate credit. Contents may not be used for commercial purposes, or adapted, remixed, transformed or built upon. (https://creativecommons.org/licenses/by-nc-nd/4.0/)

Thieme Medical and Scientific Publishers Pvt. Ltd., A-12, 2nd Floor, Sector 2, Noida-201301 UP, India 
Visible light photoinitiation then included different dental materials such as dentine bonding agents, resin cements, glass ionomer cements, denture base polymers, and epoxy resins. Nowadays, photopolymerization is being used to prepare hydrogels, three-dimensional (3D) printed and 3D bioprinted scaffolds, for tissue engineering applications, such as bone and cartilage regeneration.

A PI is a synthetic or naturally derived substance with a chromophore group that absorbs light and takes part in initiation of polymerization reaction, alone or through primary or subsequent reactions, involving one or more additional compounds such as coinitiators and accelerators. Thus a photoinitiating system (PIS) can be a one-component system with the PI only, or a two-component system with the PI and one additive mainly a coinitiator, or a three-component system with the PI plus a coinitiator and an accelerator. ${ }^{5}$

PIs can either be of type I or type II. Type I PIs generate initiating radicals by a unimolecular cleavage reaction, while type II PIs undergo bimolecular hydrogen abstraction reactions by the transfer of a hydrogen atom from a second compound, which is the coinitiator. The generation of radicals from type II PIs is slower in comparison to type I PIs because this initiation is based on a bimolecular reaction; ${ }^{6}$ thus accelerators are used with type II to enhance polymerization kinetics and lower the irradiation timec Synthetic PIs have several drawbacks such as limited solubility in water, high cost of synthesis, and cytotoxicity. ${ }^{8}$ On the contrary, naturally derived (biosourced) PIs are highly soluble in water, biocompatible, abundant, and of lower cost.

The most common mechanisms of photoinitiated polymerization used in dental and biomaterials applications are free radical or ionic polymerization. In free radical polymerization, upon light irradiation, the PI generates active free radicals that attack the monomer and convert it into a free radical that propagates the reaction and is terminated by either reaction of two free radicals (coupling) or by hydrogen transfer from a free radical to another. Photoinitiated ionic polymerization is similar to free radical polymerization but is characterized by the propagation of anionic or cationic species. Upon light irradiation, PIs produce an electron-donating compound (cationic polymerization) or an electron-withdrawing compound (anionic polymerization). Ions attack the monomer and propagation occurs by the addition of monomers to the initial ion pair. Termination of polymerization can occur by the transfer of the ion pair to a monomer or a polymer or combination with a counter ion. ${ }^{9}$

Three-dimensional printing is an additive manufacturing technology that allows fabrication of dental materials and biomaterials by adding successive layers of materials (e.g., polymers) on top of each other through computer-aided design. Photochemistry-based 3D printing uses monomers and PIs either by free radical or ionic polymerization..$^{10}$ Also, 3D bioprinting is based on deposition of biomaterials, either encapsulating cells or loaded with cells, for tissue engineering applications. Thus, using biocompatible monomers and PIs by visible light stereolithography techniques to prepare 3D bioprinted biomaterials is crucial for cell viability. ${ }^{11}$
Naturally derived PIs have been used to prepare experimental dentine bonding agents, dentine primers, methacrylates, photo-crosslinked hydrogels for tissue engineering applications, antibacterial coatings, guided tissue regeneration membranes, 3D printed and 3D bioprinted biomaterials (-Fig. 1).

An electronic search was done using MEDLINE/PubMed and Scopus databases using the keywords naturally derived, bio-sourced, photoinitiators, dental, biomaterials, 3D printing, and 3D bioprinting, and using operators or modifiers such as AND, NOT, and OR with the different keywords to review the potential naturally derived PIs for dental and biomaterials applications. Articles were chosen according to the following inclusion criteria: full text available or obtained in English language and the applications are for either dental or biomaterials uses. Structure of each naturally derived PI is shown in ( $\mathbf{- F i g . ~ 2 )}$ and the type, color, and uses of each are summarized in - Table 1.

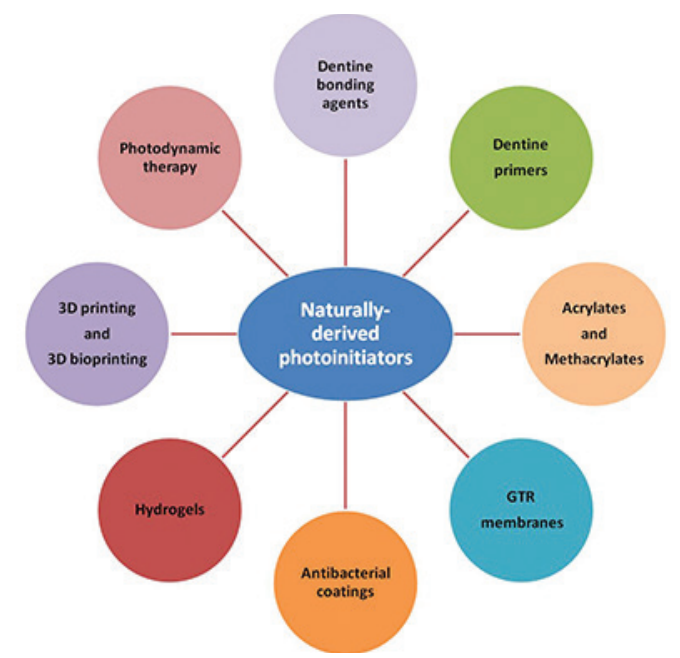

Fig. 1 Uses of naturally derived photoinitiators for dental and biomaterials applications.

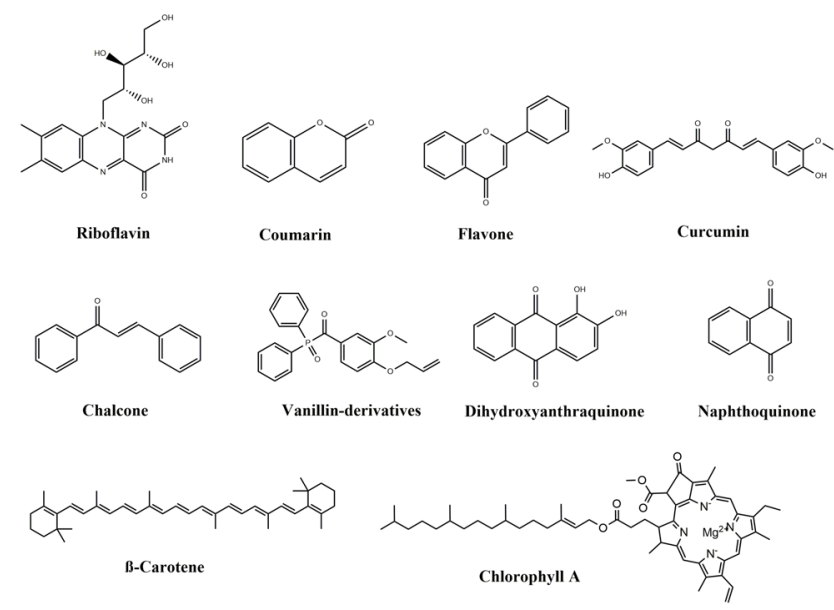

Fig. 2 Structure of naturally derived photoinitiators for dental and biomaterials applications. 
Table 1 Summary of types, color, and potential uses of naturally derived photoinitiators for dental and biomaterials applications

\begin{tabular}{|c|c|c|c|}
\hline Naturally derived photoinitiator & Type & Color & Uses \\
\hline \multirow[t]{3}{*}{ Riboflavin } & \multirow[t]{3}{*}{ I or II } & \multirow[t]{3}{*}{ Orange-yellow } & - Dentine primers \\
\hline & & & - Hydrogels \\
\hline & & & - 3D printing/bioprinting \\
\hline \multirow[t]{3}{*}{ Coumarins } & \multirow[t]{3}{*}{ II } & \multirow[t]{3}{*}{ Colorless or white } & $\begin{array}{l}\text { - Polymerization of acrylates, methacrylates, and } \\
\text { epoxy resin }\end{array}$ \\
\hline & & & - 3D printing \\
\hline & & & - Dentine bonding agents \\
\hline \multirow[t]{2}{*}{ Flavones } & \multirow[t]{2}{*}{ II } & \multirow[t]{2}{*}{ Colorless } & - Polymerization of methacrylates \\
\hline & & & - 3D printing \\
\hline \multirow[t]{3}{*}{ Curcumin } & \multirow[t]{3}{*}{ II } & \multirow[t]{3}{*}{ Orange-yellow } & - Anticariogenic photodynamic therapy \\
\hline & & & - Polymerization of methacrylates \\
\hline & & & - Antibacterial coatings \\
\hline \multirow[t]{2}{*}{ Chalcones } & \multirow[t]{2}{*}{ II } & \multirow[t]{2}{*}{ Yellow } & - Polymerization of acrylates and epoxy \\
\hline & & & - 3D printing \\
\hline \multirow[t]{2}{*}{ Vanillin derivatives } & \multirow[t]{2}{*}{ I } & \multirow[t]{2}{*}{ White } & - Polymerization of acrylates \\
\hline & & & - 3D printing \\
\hline Dihydroxyanthraquinone & II & Orange & - Polymerization of methacrylates and epoxy \\
\hline Naphthoquinones & I or II & $\begin{array}{l}\text { Yellow, red, orange, } \\
\text { purple }\end{array}$ & - Polymerization of acrylates \\
\hline$\beta$-Carotene & $\|$ & Red-orange & - Antibacterial coatings \\
\hline \multirow[t]{2}{*}{ Chlorophylls } & \multirow[t]{2}{*}{ II } & \multirow[t]{2}{*}{ Green } & - Photodynamic therapy disinfection in endodontics \\
\hline & & & - Polymerization of MMA \\
\hline
\end{tabular}

Abbreviations: 3D, three-dimensional; MMA, methyl methacrylate.

\section{Riboflavin (Vitamin B2)}

Riboflavin, or vitamin B2, is derived from plant origins, such as asparagus, broccoli, and spinach, or animal origins such as kidneys and liver. Riboflavin is important for energy production, enzyme function, and normal fatty acid and amino acid synthesis. ${ }^{12}$ It is orange-yellow in color and absorbs light in the range of 200 to $470 \mathrm{~nm}$, that is, in the UV and visible light ranges, with maximum absorption ( $\lambda \max$ ) at $223 \mathrm{~nm}$, $267 \mathrm{~nm}$, and $373 \mathrm{~nm}$ in the UV region, and $444 \mathrm{~nm}$ in the visible light region. ${ }^{13}$ Riboflavin has the ability to produce superoxide radicals that can consequently initiate a polymerization reaction. ${ }^{14}$ When used with UV, riboflavin acts as a type I PI while when used with visible light, it acts as a type II PI; thus a coinitiator is needed as a proton donor.

Riboflavin is the most commonly used naturally derived PIs in dental and biomaterials applications. Riboflavin has been used either with UVA (315-400 nm) or visible light as a primer to cross-link dentinal collagen before the application of dentine bonding agents to increase the bond strength and decrease nanoleakage. Application of riboflavin also inactivates matrix metalloproteinase (MMP) through direct cross-linking of MMPs, thus preventing degradation of the hybrid layer. ${ }^{15}$ Riboflavin has also been used to prepare photo-crosslinked membranes for guided tissue regeneration, ${ }^{16}$ hydrogels for bone, ${ }^{17}$ and cartilage regeneration. ${ }^{18}$ It is also used in corneal collagen cross-linking to treat keratoconus, using UVA light. ${ }^{19}$
Riboflavin has also been used in 3D printing of polyethylene glycol diacrylate for the potential use in tissue engineering applications. ${ }^{20,21}$ It has also been used in the 3D bioprinting of collagen/chondrocytes bioinks using extrusion-based bioprinting and visible blue light. ${ }^{22}$ A drawback of riboflavin as a PI is its orange-yellow color that might change the color of the final photo-crosslinked material.

Recently, riboflavin has been used with UV, using photodynamic therapy to reduce the count of severe acute respiratory syndrome coronavirus 2 (SARS-CoV-2), the causative agent for coronavirus disease 2019, while maintaining blood product quality. ${ }^{23}$

\section{Coumarins}

Coumarins and keto-coumarins are naturally derived compound of plant origin, especially tonka beans, strawberries, cherries, apricots, cinnamon, and vanilla grass. They possess antimicrobial, antioxidant, anticoagulant, and anti-inflammatory properties. ${ }^{24}$ They are colorless or white solids that absorb light in the near UV and visible light spectra $(270-510 \mathrm{~nm})$ with the $(\lambda \max )$ of some types at $405 \mathrm{~nm}$ and $421 \mathrm{~nm}$. Coumarins are type II PIs that can initiate the free radical polymerization of methacrylates such as bisphenol-A-glycidyl methacrylate and triethylene glycol dimethacrylate (Bis-GMA/TEGDMA) and cationic polymerization of epoxy resins. Also, they can be used to 
photopolymerize photosensitive 3D printing resins and epoxy silicones using laser diode irradiation at $405 \mathrm{~nm}$ and hydrogels for tissue engineering applications. ${ }^{25-27}$

Being colorless or white gives coumarin an advantage over other colored naturally derived PIs, as it can be used easily without changing the color of the material to be photopolymerized.

Coumarin-based iodonium hexafluoroantimonate is a white-colored PI with a light absorption spectrum near blue light ( $\lambda \max$ at $347 \mathrm{~nm}$ ) and has been used in the photopolymerization of experimental dental adhesive resin based on Bis-GMA, TEGDMA, and hydroxyethyl methacrylate, both in presence of solvent and acid monomer content. ${ }^{28}$ Coumarin oxime esters have strong absorption in the range of 400 to $480 \mathrm{~nm}$ and can initiate free radical polymerization of acrylates. ${ }^{29}$ High concentration of coumarin can be hepatotoxic, ${ }^{30}$ thus care should be taken to use the least efficient amount to photoinitiate polymerization reaction.

\section{Flavones}

Flavones are colorless naturally derived compounds that belong to flavonoids and are derived from food sources such as spices and red-purple fruits and vegetables and have antioxidant, anti-inflammatory, antimicrobial, and anticancer properties. ${ }^{31}$ Certain types of flavones have proven their antibacterial activity against cariogenic bacteria. ${ }^{32}$ They can be used as PIs using near UV and visible light irradiation (350-470 nm) with $\lambda$ max at $405 \mathrm{~nm}$. The family of flavones includes compounds such as 3-hydroxyflavone, 6-hydroxyflavone, 7-hydroxyflavone, chrysin, and myricetin. Flavones are type II PIs that are used with coinitiators and accelerators to enhance photopolymerization. Flavones can be used to photopolymerize Bis-GMA/TEGDMA composites and 3D printed biomaterials. ${ }^{8,33}$

\section{Curcumin}

Curcumin is a yellow-orange natural dye derived from the rhizomes of Curcuma longa. It has anti-inflammatory, antibacterial, and antioxidant properties, ${ }^{34}$ and antiviral properties against several viruses among which is SARSCoV-2. ${ }^{35}$ Curcumin has been proven to reduce dental bacterial biofilm formation, ${ }^{36}$ and photodynamic therapy using blue visible light and curcumin as a photosensitizer has led to $99.99 \%$ reduction in the bacterial count of Streptococcus mutans and Lactobacillus acidophilus. ${ }^{37}$ It has also proven to be efficient in the treatment of chronic gingivitis. ${ }^{38}$

It is a panchromatic PI that absorbs light in the UV-visible light (blue, green, yellow, red, and warm white) range (350-750 nm), with an intense light absorption maximum ( $\lambda$ max) at $417 \mathrm{~nm}$. It is a type II PI that is used with coinitiators and accelerators in three-component PIS for free radical polymerization of methacrylates such as urethane dimethacrylate and BIS-GMA, leading to highly cross-linked polymers with high thermal stability and mechanical strength. ${ }^{39-41}$
Curcumin has been used to prepare visible light photoinduced stainless steel antibacterial coatings against Escherichia coli and Staphylococcus aureus, with the ability to reduce bacteria by $99 \%$ and $95 \%$, respectively ${ }^{42}$ The drawback of curcumin is its yellow-orange color, which may limit its application, especially in dental applications.

\section{Chalcones}

Chalcones are a class of natural compounds that belong to flavonoids and are present in many edible plants such as fruits (tomatoes, apples, citrus), nuts, and vegetables (potatoes, shallots, bean sprouts) with anti-inflammatory, antibacterial, antiviral, anticancer, and antioxidant properties. ${ }^{43}$ Chalcone derivatives have been proven to promote osteogenic differentiation of cells, ${ }^{44}$ thus are candidates to be used in photo-crosslinked hydrogels for bone regeneration. They have also proven to have inhibitory effects against $S$. mutans cariogenic bacteria. ${ }^{44}$

Chalcones are yellow colored and are type II PIs that absorb light in the near UV and visible light region with $\lambda$ max of some types at $423 \mathrm{~nm}, 363 \mathrm{~nm}, 362 \mathrm{~nm}$, and $344 \mathrm{~nm}$. Chalcones can be used in the photopolymerization of acrylates by free radical polymerization, cationic polymerization of epoxy, and preparation of 3D printed biomaterials for tissue engineering applications. ${ }^{45,46}$

\section{Vanillin Derivatives}

(4-Allyloxy-3-methoxybenzoyl) diphenylphosphine oxide is a vanillin-derived type I PI that is white in color and can be used to induce free radical polymerization of acrylates using UV light as it absorbs light in the UV region $(385 \mathrm{~nm}){ }^{47}$ Vanillin-derived PIs have the potential to be used in the photopolymerization of 3D printed scaffolds for tissue engineering applications. Vanillin derivatives are known to possess antimicrobial properties. ${ }^{48}$ Being of type I, vanillin-derived PIs can be used without coinitiators and accelerators, thus the biocompatibility of the PIS increases.

\section{Dihydroxyanthraquinones}

Dihydroxyanthraquinone derivatives are naturally derived dyes of plant origin and are orange in color with anticancer properties. They are blue-light-sensitive with $\lambda$ max of some types at $477 \mathrm{~nm}, 417 \mathrm{~nm}$, and $426 \mathrm{~nm}$. They are type II PIs and have the potential to initiate free radical polymerization of methacrylates (Bis-GMA/TEGDMA) and cationic polymerization of epoxy. ${ }^{49}$

\section{Naphthoquinones}

Naphthoquinone derivatives, such as 5-hydroxy-1,4-naphthoquinone, are naturally derived PIs of plants, microbes, and marine origins, ${ }^{50}$ and possess antibacterial properties. ${ }^{51}$ They are yellow, red, orange, or purple in color and show maximum absorption ( $\lambda$ max) at $420 \mathrm{~nm}$ in visible blue light region. They are type II PIs that can initiate the free radical 
polymerization of acrylates.52,53 1,4-Naphthoquinone-2,3dithiol can be used as a type I PI using visible light $(478 \mathrm{~nm})$ to initiate the free radical polymerization of acrylates. ${ }^{54}$

\section{B-Carotene (Provitamin A)}

$\beta$-Carotene is a naturally derived PI that is commonly synthesized by plants, fungi, and photosynthetic bacteria. It is red-orange in color and absorbs light between 400 and $500 \mathrm{~nm}$ with maximum absorption at $450 \mathrm{~nm}$. It has photobactericidal, antioxidant properties and is a precursor of vitamin A. ${ }^{55}$ It is a type II PI that has been used in the cationic photopolymerization of limonene-derived polymer network with addition of eugenol, to prepare antibacterial coatings. ${ }^{56}$

\section{Chlorophylls}

Chlorophyll and its derivatives are naturally derived green-colored PIs that are found in plants, eukaryotes, and cyanobacteria, and are essential for photosynthesis process. It has proven antimicrobial activity against Candida albicans and Enterococcus faecalis ${ }^{57}$ and can be used as a photosensitizer in root canal disinfection, using photodynamic therapy. ${ }^{58}$ Chlorophyll derivatives have proven antiviral activity against SARS-CoV-2 virus. ${ }^{59}$ Chlorophyll A absorbs light in the violet-blue region (430-450 nm) and is a type II PI that can initiate the free radical photopolymerization of methyl methacrylate, to produce cross-linked polymethyl methacrylate. ${ }^{60}$

\section{Conclusions}

Many naturally derived PIs have proven their ability to be used as biocompatible alternatives to synthetic PIs for dental and biomaterials applications. There are a variety of naturally derived PIs with various colors and absorption spectra to choose from, according to the intended application. Most of naturally derived PIs can be used with modern conventional dental light curing units, making them applicable for experimental studies for different dental and biomaterials applications. Due to their biocompatibility and availability it is expected that in the upcoming years, research on naturally derived PIs and their dental and biomaterials applications will increase especially in the growing field of 3D bioprinting in which cell viability is essential.

\section{Conflict of Interest}

None declared.

\section{References}

1 Tasdelen MA, Lalevée J, Yagci Y. Photoinduced free radical promoted cationic polymerization 40 years after its discovery. Polym Chem 2020;11(6):1111-1121

2 Buonocore M. Adhesive sealing of pits and fissures for caries prevention, with use of ultraviolet light. J Am Dent Assoc 1970;80(2):324-330
3 Rueggeberg FA, Giannini M, Arrais CAG, Price RBT. Light curing in dentistry and clinical implications: a literature review. Braz Oral Res 2017;31(suppl 1) :e61

4 Bassiouny MA, Grant AA. A visible light-cured composite restorative. Clinical open assessment. Br Dent J 1978;145(11): 327-330

5 Xiao P, Zhang J, Dumur F, et al. Visible light sensitive photoinitiating systems: recent progress in cationic and radical photopolymerization reactions under soft conditions. Prog Polym Sci 2015;41:32-66

6 Tasdelen MA, Demirel AL, Yagci Y. Poly (propylene imine) dendrimers as hydrogen donor in Type II photoinitiated free radical polymerization. Eur Polym J 2007;43(10):4423-4430

$7 \mathrm{Kim}$ D, Scranton A. The role of diphenyl iodonium salt (DPI) in three-component photoinitiator systems containing methylene blue (MB) and an electron donor. J Polym Sci A: Polym Chem 2004;42(23):5863-5871

8 Al Mousawi A, Garra P, Schmitt M, et al. 3-hydroxyflavone and $\mathrm{N}$-phenylglycine in high performance photoinitiating systems for 3D printing and photocomposites synthesis. Macromolecules 2018;51(12):4633-4641

9 FisherJP, Dean D, Engel PS, Mikos AG. Photoinitiated polymerization of biomaterials. Annu Rev Mater Res 2001;31(1):171-181

10 Zhang J, Xiao P. 3D printing of photopolymers. Polym Chem 2018;9(13):1530-1540

11 Derakhshanfar S, Mbeleck R, Xu K, Zhang X, Zhong W, Xing M. 3D bioprinting for biomedical devices and tissue engineering: a review of recent trends and advances. Bioact Mater 2018;3(2):144-156

12 Pinto JT, Zempleni J. Riboflavin. Adv Nutr 2016;7(5):973-975

$13 \mathrm{Kim} \mathrm{SH}$, Chu CC. Visible light induced dextran-methacrylate hydrogel formation using (-)-riboflavin vitamin B2 as a photoinitiator and L-arginine as a co-initiator. Fibers Polym 2009;10(1):14-20

14 Huang R, Choe E, Min DB. Kinetics for singlet oxygen formation by riboflavin photosensitization and the reaction between riboflavin and singlet oxygen. J Food Sci 2004;69:726-732

15 Cova A, Breschi L, Nato F, et al. Effect of UVA-activated riboflavin on dentin bonding. J Dent Res 2011;90(12):1439-1445

16 Chichiricco PM, Riva R, Thomassin JM, et al. In situ photochemical crosslinking of hydrogel membrane for guided tissue regeneration. Dent Mater 2018;34(12):1769-1782

17 Arakawa C, Ng R, Tan S, Kim S, Wu B, Lee M. Photopolymerizable chitosan-collagen hydrogels for bone tissue engineering. J Tissue Eng Regen Med 2017;11(1):164-174

18 Heo J, Koh RH, Shim W, Kim HD, Yim HG, Hwang NS. Riboflavin-induced photo-crosslinking of collagen hydrogel and its application in meniscus tissue engineering. Drug Deliv Transl Res 2016;6(2):148-158

19 Subasinghe SK, Ogbuehi KC, Dias GJ. Current perspectives on corneal collagen crosslinking (CXL). Graefes Arch Clin Exp Ophthalmol 2018;256(8):1363-1384

20 Nguyen AK, Gittard SD, Koroleva A, et al. Two-photon polymerization of polyethylene glycol diacrylate scaffolds with riboflavin and triethanolamine used as a water-soluble photoinitiator. Regen Med 2013;8(6):725-738

21 Cristovao AF, Sousa D, Silvestre F, Ropio I, Gaspar A, Henriques C, et al. Customized tracheal design using 3D printing of a polymer hydrogel: influence of UV laser cross-linking on mechanical properties. 3D Print Med 2019;5:12

22 Diamantides N, Wang L, Pruiksma T, et al. Correlating rheological properties and printability of collagen bioinks: the effects of riboflavin photocrosslinking and $\mathrm{pH}$. Biofabrication 2017;9(3):034102

23 Ragan I, Hartson L, Pidcoke H, Bowen R, Goodrich R. Pathogen reduction of SARS-CoV-2 virus in plasma and whole blood using riboflavin and UV light. PLoS One 2020;15(5):e0233947 
24 Lončar M, Jakovljević M, Šubarić D, et al. Coumarins in food and methods of their determination. Foods 2020;9(5):645

25 Abdallah M, Dumur F, Hijazi A, et al. Keto-coumarin scaffold for photoinitiators for 3D printing and photocomposites. J Polym Sci 2020;58(8):1115-1129

26 Abdallah M, Hijazi A, Graff B, et al. Coumarin derivatives as versatile photoinitiators for 3D printing, polymerization in water and photocomposite synthesis. Polym Chem 2019;10(7):872-884

27 Abdallah M, Hijazi A, Dumur F, Lalevée J. Coumarins as powerful photosensitizers for the cationic polymerization of epoxy-silicones under near-UV and visible light and applications for 3D printing technology. Molecules 2020;25(9):2063

28 Boeira PO, Meereis CTW, Suárez CE, de Almeida SM, Piva E, da Silveira Lima G. Coumarin-based iodonium hexafluoroantimonate as an alternative photoinitiator for experimental dental adhesives resin. Appl Adhes Sci 2017;5(1):1-11

29 Qiu W, Li M, Yang Y, Li Z, Dietliker K. Cleavable coumarin-based oxime esters with terminal heterocyclic moieties: photobleachable initiators for deep photocuring under visible LED light irradiation. Polym Chem 2020;11(7):1356-1363

30 Lake BG. Coumarin metabolism, toxicity and carcinogenicity: relevance for human risk assessment. Food Chem Toxicol 1999;37(4):423-453

31 Hostetler GL, Ralston RA, Schwartz SJ. Flavones: food sources, bioavailability, metabolism, and bioactivity.AdvNutr2017;8(3): 423-435

32 Sato M, Fujiwara S, Tsuchiya $\mathrm{H}$, et al. Flavones with antibacterial activity against cariogenic bacteria. J Ethnopharmacol 1996;54(2-3):171-176

33 Al Mousawi A, Garra P, Dumur F, Graff B, Fouassier JP, Lalevée J. Flavones as natural photoinitiators for light mediated free-radical polymerization via light emitting diodes. J Polym Sci 2020;58(2):254-262

34 Hewlings SJ, Kalman DS. Curcumin: a review of its effects on human health. Foods 2017;6(10):92

35 Zahedipour F, Hosseini SA, Sathyapalan T, et al. Potential effects of curcumin in the treatment of COVID-19 infection. Phytother Res 2020;34(11):2911-2920 doi:10.1002/ptr.6738

36 Jahanizadeh S, Yazdian F, Marjani A, Omidi M, Rashedi H. Curcumin-loaded chitosan/carboxymethyl starch/montmorillonite bio-nanocomposite for reduction of dental bacterial biofilm formation. Int J Biol Macromol 2017;105(Pt 1):757-763

37 Araújo NC, Fontana CR, Bagnato VS, Gerbi ME. Photodynamic effects of curcumin against cariogenic pathogens. Photomed Laser Surg 2012;30(7):393-399

38 Muglikar S, Patil KC, Shivswami S, Hegde R. Efficacy of curcumin in the treatment of chronic gingivitis: a pilot study. Oral Health Prev Dent 2013;11(1):81-86

39 Zhao J, Lalevée J, Lu H, et al. A new role of curcumin: as a multicolor photoinitiator for polymer fabrication under household UV to red LED bulbs. Polym Chem 2015;6(28):5053-5061

40 Mishra A, Daswal S. Curcumin, a novel natural photoinitiator for the copolymerization of styrene and methylmethacrylate. J Macromol Sci A 2005;42(12):1667-1678

41 de Oliveira DSBL, de Oliveira LSB, Alarcon RT, da Cunha Holanda BB, Bannach G. Use of curcumin and glycerol as an effective photoinitiating system in the polymerization of urethane dimethacrylate. J Therm Anal Calorim 2017;128(3): 1671-1682

42 Condat M, Mazeran PE, Malval JP, et al. Photoinduced curcumin derivative-coatings with antibacterial properties. RSC Advances 2015;5(104):85214-85224
43 Rozmer Z, Perjési P. Naturally occurring chalcones and their biological activities. Phytochem Rev 2014;15(1):87-120

44 Choi D, Park JC, Lee HN, et al. In vitro osteogenic differentiation and antibacterial potentials of chalcone derivatives. Mol Pharm 2018;15(8):3197-3204

45 Chen H, Noirbent G, Sun K, et al. Photoinitiators derived from natural product scaffolds: monochalcones in three-component photoinitiating systems and their applications in 3D printing. Polym Chem 2020;11(28):4647-4659

46 Tehfe MA, Dumur F, Xiao P, et al. Chalcone derivatives as highly versatile photoinitiators for radical, cationic, thiol-ene and IPN polymerization reactions upon exposure to visible light. Polym Chem 2014;5(2):382-390

47 Breloy L, Negrell C, Mora AS, et al. Vanillin derivative as performing type I photoinitiator. Eur Polym J 2020;132:109727

48 Yadav R, Saini D, Yadav D. Synthesis and evaluation of vanillin derivatives as antimicrobial agents. Turk J Pharm Sci 2018;15(1):57-62

49 Zhang J, Lalevée J, Zhao J, Graff B, Stenzel MH, Xiao P. Dihydroxyanthraquinone derivatives: natural dyes as blue-light-sensitive versatile photoinitiators of photopolymerization. Polym Chem 2016;7(47):7316-7324

50 Widhalm JR, Rhodes D. Biosynthesis and molecular actions of specialized 1,4-naphthoquinone natural products produced by horticultural plants. Hortic Res 2016;3(1):16046

51 Halicki PCB, Ferreira LA, De Moura KCG, et al. Naphthoquinone derivatives as scaffold to develop new drugs for tuberculosis treatment. Front Microbiol 2018;9:673

52 Peng X, Zhu D, Xiao P. Naphthoquinone derivatives: naturally derived molecules as blue-light-sensitive photoinitiators of photopolymerization. Eur Polym J 2020;127:109569

53 Szymczak AM, Podsiadły R, Podemska K, Sokołowska J. Dyes based on a 1,4-naphthoquinone skeleton as new type II photoinitiators for radical polymerisation. Color Technol 2013; 129(4):284-288

54 Strzelczyk R, Podsiadły R. Derivatives of 1,4-naphthoquinone as visible-light-absorbing one-component photoinitiators for radical polymerisation. Color Technol 2015;131(3): 229-235

55 Grune T,LietzG,Palou A, etal.Beta-carotene is an importantvitamin A source for humans. J Nutr 2010;140(12):2268S-2285S

56 Breloy L, Ouarabi CA, Brosseau A, et al. $\beta$-carotene/limonene derivatives/eugenol: green synthesis of antibacterial coatings under visible-light exposure. ACS Sustain Chem\& Eng 2019;7(24):19591-19604

57 Maekawa LE, Lamping R, Marcacci S. Maekawa MY, Nassri MRG, Koga-Ito CY. Antimicrobial activity of chlorophyll-based solution on Candida albicans and Enterococcus faecalis. Rev Sul Bras Odontol 2007;4(2):36-40

58 Diogo P, Mota M, Fernandes C, et al. Is the chlorophyll derivative $\mathrm{Zn}(\mathrm{II}) \mathrm{e}$ Me a good photosensitizer to be used in root canal disinfection? Photodiagn Photodyn Ther 2018;22:205-211

59 Clark NF, Taylor-Robinson AW. COVID-19 therapy: could a chlorophyll derivative promote cellular accumulation of Zn2+ions to inhibit SARS-CoV-2 RNA synthesis? Front Plant Sci 2020;11:1270

60 Wang GX, He JY, Liu LC, Wu H, Zhong M. Photo-induced atom transfer radical polymerization of MMA with chlorophyll A as photoinitiator. J Polym Res 2016;23(5):101 